5668. Xanthonia 10-notata Say.

5672. Heteraspis marcassita Germ.

5689. Chrysochus cobaltinus Lec.

5719b. Colapsis puncticollis Say.

- Chalcophana placida -

5733. Chrysomela disrupta Rog.

5837. Trirhabda convergens Lec.

5886. Graptodera foliacea Lec.

- - Graptodera bimarginata Say.

6124. Coniontis ovalis Esch.

6202. Iphthimus serratus Mannh.

6357. Helops convexulus Lec.

6374. Hymenorus pilosus Mels.

6450. Notoxa anchora Hentz.

-. Pentaria rugicollis Lec.

6578. Anaspis nigra Hald,

6583. Anaspis rufa Say.

6593. Mordella melana Germ.

6594. Mordella scutellaris Fab.

6731. Epicauta ferruginea Say.

6809. Nemognatha lurida Lec.

6825. Nemognatha immaculata Say.

- Mycterus n. sp.

6874. Crymodes discicollis Say.

7017. Lepyrus geminatus Say.

- - Magdalis Le Contei —.

7106. Malaninus nasicus Say.

- Anthonomus squamosus Lec.

7322. Pityopthorus retusus Lec.

- Pityophthorus sp.

7364. Tomicus pini Say.

Number of species, 99 .

\title{
ADDITIONS TO THE CATALOGUE OF KANSAS COLEOPTERA.
}

\author{
By E. A. Popenoe, Topeka.
}

The following list of additions to the Catalogue of Kansas Coleoptera includes in great part the results of the collections of 1877 and 1878, made in different localities by Prof. F. H. Snow and the writer. The species were determined by Dr. John L. Le Conte, of Philadelphia, to whom thanks are due for many favors. Those species noted from Lawrence are added by Prof. Snow. The additions number 435, increas ing the list total to 1711 species.

49. Cicindela lepida Dej. Lawrence; taken by A. E. Blood.

67. Cicindela Pilatii Guer. Lawrence; taken by C. B. Cramer. 
221. Dyschirius haemorrhoidalis Dej. Topeka; common in mud on the creek banks.

233. Dyschirius filiformis Lec. Lawrence.

242. D. hispidus Lec. Less common than No. 221; found in the same localities.

246. Ardistomis virides Say. Topeka and Lawrence.

252. Clivina impressifrons Lec. Lawrence.

261. Clivina Americana Dej. Lawrence.

266. Clivina ferrea Lec. Lawrence.

-_. Clivina new species. Topeka.

292. Brachynus alternans Dej. Lawrence.

296. Brachynus fumans Fab., var. similes Lec. Wallace county (Snow).

300. Brachynus stygicornis Say. Western Kansas.

309. Micrixys distinctus Hald. Peabody, from C. P. Arbuthnot.

352. Lebia viridipennis Dej. Topeka and Lawrence.

364. Aphelogenia fuscata Lec. Lawrence (Snow), and West Kansas (Cooper).

386. Axinopalpus biplagiatus Dej. Gove county (Snow).

393. Glycia viridicollis Lec. Lawrence, and Gove county.

448. Platynus pusillus Lec. Lawrence.

461b. Platynus viridis Lec. Topeka.

484. Platynus rubripes Zimm. Lawrence.

501. Platynus sordens Kby. Topeka; rare.

503. Platynus ruficornis Lec. Lawrence.

- Platymus cinctus. Lawrence.

- Platynus new species.

563. Evarthrus incisus Lec.

618. Pterostichus scitulus Lec. Western Kansas.

619. Pterostichus latulus Lec. Lawrence.

626. Pterostichus lucublandus, var. bicolor Lec. Topeka; two specimens; also at Lawrence.

643a. Pterostichus desidiosus Lec. Lawrence.

672. Amara laticollis Lec. Topeka.

693. Amara basilaris Say. Lawrence.

703. Amara convexa Lec. Wallace county (Snow).

709. Amara obesa Say. Western Kansas.

710. Amara diffinis Lec. Wallace county (Snow).

712. Amara chalcea Dej. Western Kansas.

717. Amara gibba Lec. Topeka and Lawrence.

-_. Amara fulvipes Putz. Topeka; one specimen; also at Lawrence.

-- Amara cupreolata Putz. Topeka and Lawrence.

726. Badister pulchellus Lec. Lawrence.

729. Badister micans Lec. Lawrence.

748. Dicclus furvus Say. Topeka; a single specimen taken.

794. Chlcnius purpuricollis Randall. Lawrence.

796. Atranus pubescens Dej. Topeka; two specimens.

801. Oodes amaroides Dej. Lawrence.

827. Agonoderus partiarius Say. Topeka.

851. Anisodactylus Harrisii Lec. Lawrence.

859. Anisodactylus discoideus Dej. Lawrence.

-_. Anisodactylus agricola Say. Topeka; frequent.

913. Selenophoris opalinus Lec. Lawrence.

916. Selenophorus pedicularis Dej. Wallace county (Snow). 
921. Selenophorus ellipticus Dej. Lawrence.

942. Haipalus ventralis Lec. Wallace county (Snow).

-_. Stenolophus new species. Topeka; one specimen.

975. Stenolophus plebeius, var. fuscatus Dej. Lawrence.

1058. Bembidium perspicuum Lec.

1063. Bembidium striola Lec. Lawrence.

1096. Bembidium variegatum Say.

1099. Bembidium versicolor Lec.

1110. Bembidium affine Say. Topeka and Lawrence.

1127. Tachys proximus Say. Lawrence.

1154. Tachys ferrugineus Dej. Topeka; one specimen.

1158. Tachys nebulosus Chd. Topeka; one specimen.

1188. Hydrovatus cuspidatus Germ. Lawrence.

1201. Hydroporus dissimilis Harris. Lawrence.

1208. Hydroporus patruelis Lec. Lawrence, and Gove county.

1217. Hydroporus lacustris Say. Topeka; abundant where found.

1236. Hydroporus undulatus Say. Gove county (Snow).

1262. Hydroporus concinnus Lec. Topeka; not rare.

1283. Laccophilus 4-lineatus Horn. Gove county (Snow).

1291. Thermonectes ornaticollis Aube. West Kansas ; also at Lawrence.

1291a. Thermonectes nigrofasciatus Aube. Topeka.

1322. Rhantus notatus Fab. Lawrence.

1326. Rhantus calidus Fab.

1347. Gaurodytes disintegratus Cr. Lawrence.

1349. Gaurodytes semivittatus Lec.

1356. Gaurodytes obliteratas Lec. Lawrence.

1365. Gaurodytes (near) parallelus Lec. Lawrence.

1400. Gyrinus maculiventris Lec.

1402. Gyrinus parcus Say. Western Kansas (Cooper and Snow).

1454. Tropisternus sublavis Lec. Western Kansas (Cooper and Snow).

1465. Berosus miles Lec. Gove county (Snow).

1471. Berosus pantherinus Lec. Lawrence.

1472. Berosus peregrinus Hb.

1489. Philhydrus nebulosus Say.

1493. Philhydrus ochraceus Mels. Topeka and Lawrence; rare.

1494. Philhydrus reflexipennis Zimm (?).

1504. Philhydrus fimbriatus Mels. Gove county (S. W. Williston).

1516. Hydrobius subcupreus Say. Gove county (Snow).

1534. Cercyon protextum Say. Topeka; not rare, and found under decaying vegetable refuse.

- Trichopteryx sp. Lawrence.

1710. Tachyporus nanus Er. Lawrence.

1763. Quedius capucinus Grav. Lawrence.

1780. Staphylinus exulans Er. Western Kansas.

1787. Staphylinus cinnamopterus Grav. Lawrence.

1799. Ocypus ater Grav. Lawrence.

1803. Philonthus aneus Rossi. Lawrence.

1821. Philonthus lomatus Er. Topeka and Lawrence.

1834. Philonthus apicalis Say. Lawrence.

1835. Philonthus sobrinus Er. Topeka and Lawrence.

Philonthus fusiformis Fauvel Ms. Gove county (Snow). 
- Philonthus sp. Two undescribed species. Lawrence.

--_. Xantholinus fusciceps Fauvel. Lawrence.

1880. Lathrobium collare Er. Lawrence.

1881. Lathrobium dimidiatum Say.

1894. Stilicus tristis Mels. Lawrence.

-_. Bledius confusus - - Lawrence.

1991. Oxytelus fuscipennis Mann. Lawrence.

1995. Oxytelus nıtidulus (?) Grav. Lawrence.

2001. Apocellus sphcericollıs Say. Lawrence.

2012. Anthophagus verticalis Say. Lawrence.

2019. Acidota subcarinata Er. Lawrence.

2046. Omalium repandum Er. Lawrence.

2095. Ctenistes piceus Lec. Topeka.

2100. Tymus humeralis Aubé. Topeka.

2103. Tychus longipalpus Lec. Topeka.

2124. Bryaxis rubicunda Aubé. Topeka.

2149. Batrısus globosus Lec. Lawrence.

2175. Silpha Carolina L. Wallace county (Snow).

-. Agathidium sp. Gove county (Snow).

2312. Sericoderus flavidus Lec. Lawrence.

2335. Scaphrsoma suturale Lec. Topeka.

2336. Scaphisoma terminatum Mels. Topeka.

2340. Toxidium compressum Zimm. Lawrence.

2417. Trogoderma pallipes Ziegl. Lawrence.

2426. Cryptorhopalum hamorrhoidale Lec. Lawrence.

2441. Mycetina morosa Lec. West Kansas (Cooper).

2468. Litargus sexpunctatus Say. Topeka and Lawrence; frequent.

- Ceracis sp. Lawrence.

2504. Ceracis Sallei Mell. Topeka; in fungi; common

2511. Languria lata Lec. Gove county (Snow).

2514. Languria gracılis Newm. Gove county (Snow); Tonganoxie (T. B. Ashton).

- Atomaria new species. Topeka and Lawrence.

2581. Silvanus bidentatus Fabr. Topeka.

2590. Telephanus velox Hald. Topeka ; one specimen.

2599. Lamophlaus fasciatus Mels. Topeka.

2601. Lamophlaus testaceus Fab. Topeka.

2605. Lamophlaeus adustus Lec. Topeka.

2666. Bactridium nanum Er. Lawrence.

2688a. Trogosita intermedia Horn. Topeka.

2688b. Trogosita dubia Horn. Lawrence.

2717. Colastus semitectus Say. Topeka; under the bark on freshly cut willow stumps, feeding on the sap.

2719. Colastus truncatus Rand. With the last named.

2725. Carpophilus niger Say. Topeka, and West Kansas.

2731. Carpophilus brachypterus Say. Topeka; very abundant in early spring (1878) on blooming willow catkins.

2743. Epurcea labilis Er. Topeka; three specimens.

2757. Lobiopa undulata Say. Topeka; four specimens, taken in July, on the ground, under decaying fruit.

2813. Olibrus semistriatus Lec. 
-. Olibrus ergoti Walsh Ms. Lawrence.

2827. Hippodamia 5-signata Kby.

2867. Psyllobora 20-maculata Say. Topeka and Lawrence.

2871. Exochomus Pilatii Muls. Lawrence.

2877a. Brachyacantha 10-pustulata Mels. Lawrence.

2877 d. Brachyacantha albifrons Say. West Kansas.

2897. Hyperaspidius trimaculata L. West Kansas.

2900. Scymnus amabilis. Lec.

2906. Scymnus terminatus Say. Lawrence.

2909. Scymnus Americanus Muls. Lawrence.

2924. Scymnus cervicalis Muls. Gove county (Snow.)

-_. Scymnus punctatus -. Lawrence.

-_. Pentilia sp. Lawrence.

2994. Stenelmis linearis Zimm. Lawrence.

2996. Stenelmis crenatus Say. West Kansas.

2999. Stenelmus vittipennis Zimm. Lawrence.

3013. Heterocerus collaris Kies. Lawrence.

3042. Hister marginicollis Lec. Lawrence.

- - Hister binotatus -. Lawrence.

3062. Hister bimarginatus Linn. Lawrence.

3115. Paromalus seminulum Er.

3158. Saprinus fimbriatus Lec. Wallace county (Snow.)

- Acritus sp. Lawrence.

3198. Aeletes politus Lec. Topeka.

3223. Canthon ebenus Say. Wallace county (Snow.)

3248. Onthophagus orpheus - West Kansas and Lawrence.

3269. Aphodius vittatus Say. West Kansas and Lawrence.

3294. Aphodius terminalis Say. Lawrence.

3338. Ochodceus musculus Say. West Kansas.

3371. Trox scutellaris Say. Lawrence.

3385. Trox erinaceus Lec. Lawrence.

3451. Diazus rudis Lec.

3459a. Diplotaxis frondicola Bl. Lawrence.

3466. Diplotaxis punctipennis Lec. Lawrence.

3468. Diplotaxis Harperi Bl. West Kansas (Cooper.)

3475. Diplotaxis morula Lec. West Kansas.

3482. Diplotaxis pacata Lec. Kansas specimens were received from Prof. F. H.

Snow.

3484. Diplotaxis Haydeni Lec. West Kansas (Cooper and Snow.)

3494. Phyllophaga longitarsis Say. West Kansas (Cooper.)

3511. Phyllophaga lugubris Lec. Topeka.

3519. Phyllophaga prunina Lec. Gove county (Snow.)

3521. Phyllophuga affinis Lec. Topeka.

3545. Phyllophaga glabricula Lec. Wallace county (Snow.)

3614. Aphonus pyriformis Lec. Wallace county (Snow.)

3615. Aphonus tridentatus Say. West Kansas.

3654. Cremastochilus saucius Lec. West Kansas (Cooper and Snow.)

3689. Dicerca prolongata Lec. Lawrence.

—. Dicerca pruinosa. Lawrence.

- C. Chrysobothrus new species.

3739. Anthaxia cyanella Gory. Lawrence. 
3742. Anthaxia quercata Fab. Lawrence.

3827. Agrilus pubiventris Cr. Gove county (Snow.)

3828. Agrilus egenus Gory. Lawrence.

3829. Agrlus lacustris Lec.

3833. Agrilus muticus Lec. West Kansas.

- Agrilus subvittatus Lec. Lawrence.

- Agrilus new species near torquatus Lec.

- Agrilus new species near mancus.

3836. Taphrocerus gracilis Say. West Kansas (Cooper); also at Lawrence.

3845. Pachyscelus laevigatus Say. Topeka; two specimens.

—. Limonius interstitialis -. Topeka.

3946. Cardiophorus cardisce Say. Lawrence.

4003. Elater nigricollis Hbst. Lawrence.

4009. Elater Sayi Lec. Lawrence.

4073. Anchastus digitatus Lec. Lawrence.

4154. Melanotus verberans Lec. Gove county (Snow.)

4159. Melanotus paganus Cand. Lawrence.

4171. Melanotus sagittarius Lec. Lawrence.

—. Limonius interstitialis —. Topeka.

4230. Athous scapularis Say. Lawrence.

4240. Athous montanus Lec. Lawrence.

-. Asaphes brevicollis -. Lawrence.

4347. Asaphes bilobatus Say. Lawrence.

4400. Prionocyphon discoideus Say. Topeka; rare.

4403. Helodes pulchella Guer. Topeka and Lawrence; rare.

4414. Eucinetus terminalis Lec. Topeka.

4448. Lucidota punctata Lec. Gove county (Snow.)

4453. Photinus nigricans Say.

4518. Telephorus dentiger Lec.

4527. Telephorus dichrous Lec.

4559. Silis percomis Say. Topeka.

4561. Ditemnus bidentatus Say. Wallace county (Snow.)

4563. Loberus abdominalis Lec. West Kansas.

- L. Loberus impressus -. Lawrence.

4589. Collops limbatus Lec. West Kansas.

4612. Anthocomus Erichsoni Lec. Lawrence.

4628. Attalus morulus Lec. Lawrence.

46r0. Pristoscelis texanus Lec. West Kansas.

4697. Elasmocerus terminatus Say. Lawrence.

4722. Clerus Spinola Lec. Gove county (Snow.)

4734. Clerus cordifer Lec. Wallace county (Snow.)

- Clerus n. sp. Gove county (Snow.)

4765. Hydnocera tabida Lec. Gove county (Snow.)

4769. Phyllobcenus dislocatus Say. Lawrence.

4778. Cregya oculata Say. Lawrence.

4789. Corynetes rufipes Fab. West Kansas; common.

4822. Oligomerus obtusus Lec. Lawrence.

4824. Sitodrepa panicea L. Found in great numbers in some grains and seeds received in exchange at the Centennial Exhibition by the State Board of Agriculture.

- Sitodrepa $\mathrm{n} \mathrm{sp.}$ 
4839. Petalium bistriatum Say.

- Hemiptychus new species.

4853. Hemiptychus gravis (?) Lec. Lawrence.

4863. Conocara oculata Say. Topeka and Lawrence; frequent on bushes and plants in the woods on Soldier creek.

4920. Prionus fissicornis Hald. Wallace county (Snow).

5001. Elaphidion rufulum Hald. Lawrence.

5015. Elaplidion unicolor Rand. Lawrence; bred from Red-bud (Cercis Canadensis) by Geo. C. Brackett, Esq.

5025. Tylonotus bimaculatus Hald. Lawrence.

5091. Crossidus pulchellus Lec. West Kansas.

5094. Crossidius discoideus Say. West Kansas.

5104. Cyllene decora Oliv. var infausta Lec. Gove county (Snow).

5291. Leptura spharicollis Say. Lawrence.

5310. Monilema semipunctatum Lec. West Kansas; common on Opuntia. (Cooper.)

5311. Monilema armatum Lec. West Kansas.

Sternidius new species. Lawrence.

5394. Eupogonius vestitus Say. Lawrence.

5427. Oberea ruficollis Fab. West Kansas.

5433a. Oberea basalis Lec. Gove county (Snow.)

5439. Oberea Schaumii Lec. var. Lawrence.

- Tetrops sp. Lawrence.

5460. Caryoborus arthriticus Fab. Lawrence.

5465. Bruchus scutellaris Fab. Lawrence.

5478. Bruchus limbatus Horn.

5481. Bruchus pruininus Horn. Gove county, from S. W. Williston.

5496. Bruchus fraterculus Horn. Topeka, and Wallace county.

- - Bruchus n. sp. Lawrence.

5514. Donacia alutacea Lec. Lawrence.

5534. Orsodachna atra Ahr. Topeka and Lawrence ; very common in spring in certain localities on the blossoms of wild plum. ' Var. vittata occurs at Lawrence.

5578. Exema conspersa Mann. Lawrence.

5578a. Exema dispar Lec. Topeka; frequent.

5587. Cryptocephalus sellatus Suffr. Lawrence.

5588. Cryptocephalus lituratus Fab.

5588a. Cryptocephalus lativittis Germ. Topeka.

5589. Cryptocephalus venustus Fab. Topeka and Lawrence.

5595. Cryptocephalus confluens Say. West Kansas.

5609. Cryptocephalus notatus Fab. Lawrence.

5613. Cryptocephalus catarius Suffr. Common on plants in woods in spring; Topeka.

5617. Cryptocephalus chlorizans Suffr. Lawrence.

- Cryptocephalus new species. With 5613, and very common.

5664. Pachybrachys hepaticus Mels. Topeka and West Kansas.

5666. Fidia murina Cr. Lawrence.

- Heteraspis new species. Topeka; two specimens.

5674. Heteraspis smaragdula Lec. West Kansas (Cooper.)

5693. Chalcoparia globosa Oliv. West Kansas (Cooper and Snow).

5695. Paria aterrima Oliv., var. canella. Lawrence.

-_. Chalcophana picipes Oliv. West Kansas (Cooper). 
5715a. Colaspis flavida Say, var. lineata. Gove county (Snow).

5730. Chrysomela conjuncta Rog. Wallace county (Snow).

5733. Chrysomela disrupta Rog. Lawrence, and Gove county.

5750. Chrysomela auripennis Say. Lawrence.

5772. Plagiodera oviformis Lec. Gove county (Snow).

- - Adimonia externa Say. Lawrence.

5756. Gastrophysa formosa Say. West Kansas.

5805. Diabrotica blandula Lec. West Kansas (Snow).

- Diabrotica new species. West Kansas.

5822. Galerucella sagittariae Gyll. Topeka; rare.

5826. Galerucella notata Fab. West Kansas.

5838. Trirhabda nitidicollis Lec. Wallace county (Snow).

5842. Hypolampsis pilosa Ill. Lawrence.

5861. Oedionychis sexmaculata Ill. Topeka; one specimen; also taken at Lawrence.

5871. Disonycha discoidea Fab. Lawrence.

5874. Disonycha collaris F. race mellicollis Say. Lawrence.

5875. Graptodera var. subplicata Lec. Topeka; very common on willows along the Kansas river.

5878. Graptodera ignita Ill. (var.) Topeka and Lawrence.

5881. Graptodera obliterata Lec. Topeka and Lawrence.

5882. Graptodera torquata Lec. West Kansas (Cooper and Williston).

-_. Graptodera bimarginata Say.

-_. Graptodera new species.

5900. Batophila cerina (?) Lec. Lawrence.

5906. Orchestris bipustulata Fab. Topeka and Lawrence; frequent.

5908. Orchestris Lewisii Cr.

5924. Lyperaltica fuscula Lec. Topeka, on composite flowers; common.

5925. Lyperaltica senilis Say. Topeka; four specimens taken on Actinomeris.

5927. Crepidodera erythropus Mels.

5928. Crepidodera var. violacea Mels. Common in spring on leaves of wild cherry (Prunus).

5928. Crepidodera var. opulenta Lec. Common on leaves of willow.

5930a. Crepidodera mancula Lec. Lawrence.

5931. Crepidodera scabricula Cr. Topeka; one specimen.

-_. Crepidodera new species. Lawrence.

5935. Epitrix fuscula Cr. Topeka; common in timber on various plants.

5937. Epitrix near hirtipennis Mels. Lawrence.

5941. Chcetocnema subviridis Lec. Topeka and Lawrence.

5947. Chotocnema confinis Cr. Lawrence.

5971. Microrhopala porcata Mels. West Kansas.

5977. Chelymorpha Lewisii Cr. West Kansas.

5980b. Cassida ellipsis Lec. West Kansas (Snow).

- C. Cassida sexpunctata - . West Kansas; frequent on Ipomca leptophylla.

6168. Eleodes fusiformis Lec. West Kansas (Cooper).

6245. Blapstinus vestitus Lec. Wallace county (Snow).

6291. Paratenetus punctatus Sol. Topeka and Lawrence.

-_. Paratenetus new species. Topeka.

- Mycetochares lugubris -. Lawrence.

6390. Isomera quadristriata Cooper. Topeka.

6456. Notoxus apicalis Lec. West Kansas. 
6458. Notoxus subtilis Lec. Wallace county (Snow).

6459. Notoxus bifasciatus Lec. Topeka.

- Notoxus n. sp. Lawrence.

6472. Anthicus elegans Laf. Lawrence.

6479. Anthicus floralis Payk. Topeka; frequent.

6501. Anthicus spretus Lec. Lawrence.

- - Anthicus n. sp. Lawrence.

6527. Xylophilus fasciatus Mels. Lawrence.

6541. Synchroa punctata Newm. Lawrence.

6560. Symphora flavicollis Hald. Topeka; rare.

6561. Symphora rugosa Hald. Lawrence.

6583. Anaspis rufa Say. Gove county (Snow).

6606. Mordella discoidea Mels. Lawrence.

6628. Mordellistena amica Lec. Lawrence.

6648. Mordellistena unicolor Lec. Wallace county and Gove county (Snow.)

6662. Mordellistena suturella Helmuth. Gove county (Snow.)

-. Mordellistena new species. Wallace county (Snow.)

- E. Epicauta trichrus var. Gove county (Snow.)

6742. Epicauta Stuarti Lec. Wallace county (Snow.)

6740. Epicauta lemniscata Fab. Lawrence.

6809. Nemognatha lurida Lec. Wallace county and Gove county (Snow.)

Gnathium new species. West Kansas.

Eugnamptus angustatus Hbst. Lawrence.

Rhynchites cyanellus Lec. Lawrence.

Rhynchites aeratus Say. Lawrence.

Rhynchites hirtus Oliv. West Kansas.

Attelabus nigripes Lec. Lawrence.

Epiccerus formidolosus Schh. Lawrence.

Eupagoderes sordidus Lec. Lawrence.

Sitones tibialis Germ. Gove county (Snow.)

Listronotus squamiger Say. Lawrence.

Listronotus caudatus Say. This species and L. nebulosus occur together on Sagittaria.

Lixus scrobicollis Schh. Lawrence.

Dorytomus brevicollis Lec. Topeka; common on willow.

Dorytomus squamosus Lec. Topeka, and Gove county.

Smicronyx tychoides Lec. Lawrence.

Smicronyx vestitus Lec. Topeka.

Smicronyx squamulatus Lec. Topeka.

Smicronyx new species. Topeka and Lawrence.

Endalus limatulus Lap. Gove county (Snow.)

Lissorhoptrus apiculatus Gyll. Lawrence.

Otidocephalus myrmex Hbst. Lawrence.

Otidocephalus Chevrolatii Horn. Topeka; three specimens.

Magdalis pandura Say. Topeka and Lawrence.

Anthonomus fulvus Lec. West Kansas.

Anthonomus rubidus Lec. Topeka.

Anthonomus squamosus Lec. West Kansas.

Anthonomus corvulus Lec. Lawrence.

Anthonomus sycophanta Walsh. Topeka.

Orchestes ephippiatus Say. Topeka and Lawrence; frequent on willow. 
Alyca ephippiata Say. Topeka and Lawrence; frequent on willows.

Thysanocnemis fraxini Lec. Lawrence.

Conotrachelus cribricollis Say.

Conotrachelus cratagi Walsh. Lawrence.

Conotrachelus new species. Topeka; one specimen.

Rhyssematus palmacollis Say. Lawrence.

Rhyssematus rqualis -.

Acalles turbidus Lec. West Kansas (Snow.)

Acalles carinatus Lec. Lawrence.

Cryptorhynchus obliquefasciatus Boh. Lawrence.

Acamptus rigidus Lec. Lawrence.

Ceutorhynchus decipiens Lec. Gove county (Snow.)

Ceutorhynchus new species. West Kansas.

Pelenomus sulcicollis Fahr. Lawrence.

Baris macer (?) Lec. Gove county (Snow.)

Baris confinis Lec. Topeka and Lawrence.

Baristransversa Lec. West Kansas.

Baris tumescens Lec. Lawrence.

Baris striata Say.

Baris new species, near carinulata.

Baris pruinosa Lec. West Kansas and Lawrence.

Ampeloglypter Sesostris Lec. Lawrence. Injures grave vines.

Microcholus new species. Topeka; two specimens.

Barilepton cribricolle Lec. Lawrence.

Barilepton quadricolle - . West Kansas.

Centrinus neglectus Lec. Lawrence.

Centrinus falsus Lec.

Centrinus prolixus - . West Kansas.

Centrinus confusus Boh.

Centrinus modestus Boh.

Sphenophorus ochreus Lec. Lawrence.

Sphenophorus aqualis Gyll.

Sphenophorus melanocephalus Fab.

Sphenophorus pertinax Oliv. Topeka and Lawrence.

Sphenophorus vomerinus Lec., var. baridioides. West Kansas (Snow.)

Sphenophorus placidus Say. Lawrence.

Hypothenemus hispidulus Lec. Topeka.

Hypothenemus distinctus Lec. Lawrence.

Micracis suturalis Lec. Lawrence.

Scolytus quadrispinosus Say. Lawrence.

Hylesinus aculeatus Say. Lawrence.

Eurymycter fasciatus Oliv. Lawrence.

Hormiscus saltator Lec. Topeka and Lawrence.

Anthribus cornutus Say. Lawrence. 


\section{$2 \mathrm{BHL}$ Biodiversity Heritage Library}

Alonzo, Popenoe Edwin. 1878. "Additions to the catalogue of Kansas

Coleoptera." Transactions of the Kansas Academy of Science 6, 77-86.

View This Item Online: https://www.biodiversitylibrary.org/item/34353

Permalink: https://www.biodiversitylibrary.org/partpdf/30460

\section{Holding Institution}

Harvard University, Museum of Comparative Zoology, Ernst Mayr Library

\section{Sponsored by}

Harvard University, Museum of Comparative Zoology, Ernst Mayr Library

\section{Copyright \& Reuse}

Copyright Status: NOT_IN_COPYRIGHT

This document was created from content at the Biodiversity Heritage Library, the world's largest open access digital library for biodiversity literature and archives. Visit BHL at https://www.biodiversitylibrary.org. 